\title{
Multiscale Control of Nanocellulose Assembly: Transferring Remarkable Nanoscale Fibril Mechanics to Macroscale Fibers
}

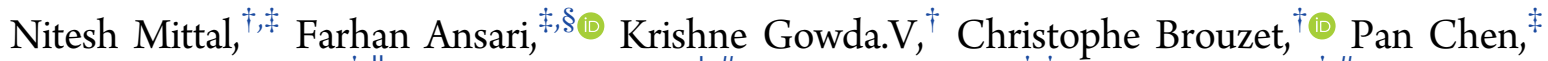

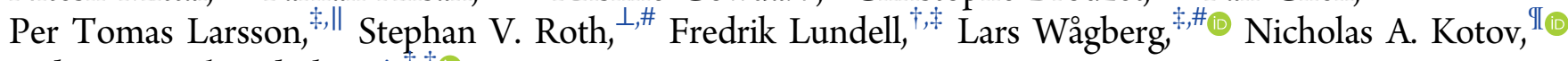
and L. Daniel Söderberg*,,$+\neq 0$

${ }^{\dagger}$ Linné FLOW Centre, KTH Mechanics, ${ }^{\ddagger}$ Wallenberg Wood Science Centre, and ${ }^{\#}$ Department of Fibre and Polymer Technology, KTH Royal Institute of Technology, SE-100 44 Stockholm, Sweden

${ }^{\S}$ Department of Materials Science and Engineering, Stanford University, Stanford, California 94305-2205, United States

"RISE Bioeconomy, P.O. Box 5604, SE-114 86 Stockholm, Sweden

${ }^{\perp}$ Deutsches Elektronen-Synchrotron (DESY), D-22607 Hamburg, Germany

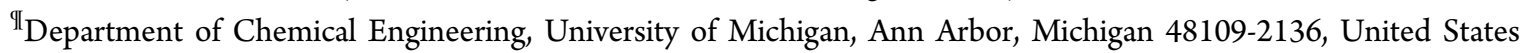

\section{Supporting Information}

ABSTRACT: Nanoscale building blocks of many materials exhibit extraordinary mechanical properties due to their defect-free molecular structure. Translation of these high mechanical properties to macroscopic materials represents a difficult materials engineering challenge due to the necessity to organize these building blocks into multiscale patterns and mitigate defects emerging at larger scales. Cellulose nanofibrils (CNFs), the most abundant structural element in living systems, has impressively high strength and stiffness, but natural or artificial cellulose composites are 3-15 times weaker than the CNFs. Here, we report the flow-assisted organization of CNFs into macroscale fibers with nearly perfect unidirectional alignment. Efficient stress transfer from macroscale to individual CNF due to cross-linking and high degree of order enables their Young's modulus to reach up to $86 \mathrm{GPa}$ and a tensile strength of 1.57 GPa, exceeding the mechanical properties of known natural or synthetic biopolymeric materials. The specific strength of our CNF fibers engineered at multiscale also exceeds that of metals, alloys, and glass fibers, enhancing the potential of sustainable

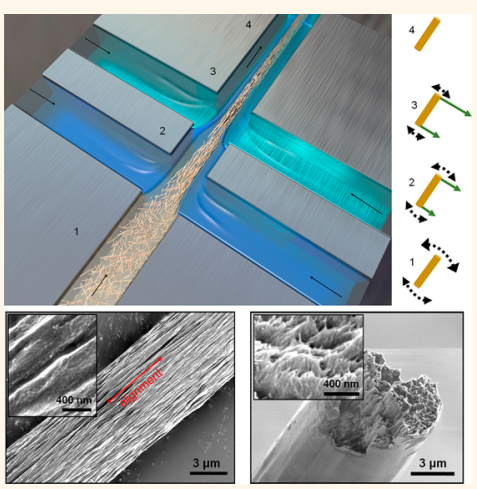
lightweight high-performance materials with multiscale self-organization.

KEYWORDS: bio-based materials, self-organization, mechanical properties, microfluidics, cellulose nanofibrils, nanocomposites

1 he quest for more eco-friendly and energy-efficient technologies accentuates the need to develop lightweight structural materials with exceptional mechanical performance from renewable resources. ${ }^{1}$ Nature has long developed abilities to tightly control the structural features of its high-performance finite size building blocks with wellordered arrangements at nano- and molecular level. ${ }^{2,3}$ Lately, scientists have been seeking ideas of mimicking natural materials' architecture based on engineering design principles, typically called "bioinspired assembly". An overarching challenge in structural materials fabrication is to translate the extraordinary mechanical properties of nanoscale building blocks (e.g., tensile strength and Young's modulus) to the macroscale bulk materials. ${ }^{4}$ This problem arises from the fundamentally nonideal stress transfer from the macro- to molecular scale that prevents efficient utilization of the high mechanical performance of nanoscale building blocks. Poor adhesion and building block misalignment creates large amount of nanoscale defects that limits the materials performance at scales most common to human technologies. ${ }^{2,3}$

The architecture of wood, especially the outer cell wall layer (S2 layer) that possesses the highest strength and stiffness among all layers, provides leads for structural design of uniaxial high-performance materials. ${ }^{5}$ The S2 layer is made of semicrystalline CNFs that are aligned and embedded in a matrix of hemicellulose and lignin to form macrofibers. Being cross-linked to hemicellulose and lignin via an abundance of carboxyl groups, ${ }^{6}$ the crystalline regions of CNF contain the backbone of cellulose molecules, which makes them stiff (Young's modulus of $130-150 \mathrm{GPa})$ and strong ( 1.0-3.0

Received: February 8, 2018

Accepted: May 2, 2018

Published: May 9, 2018 
a

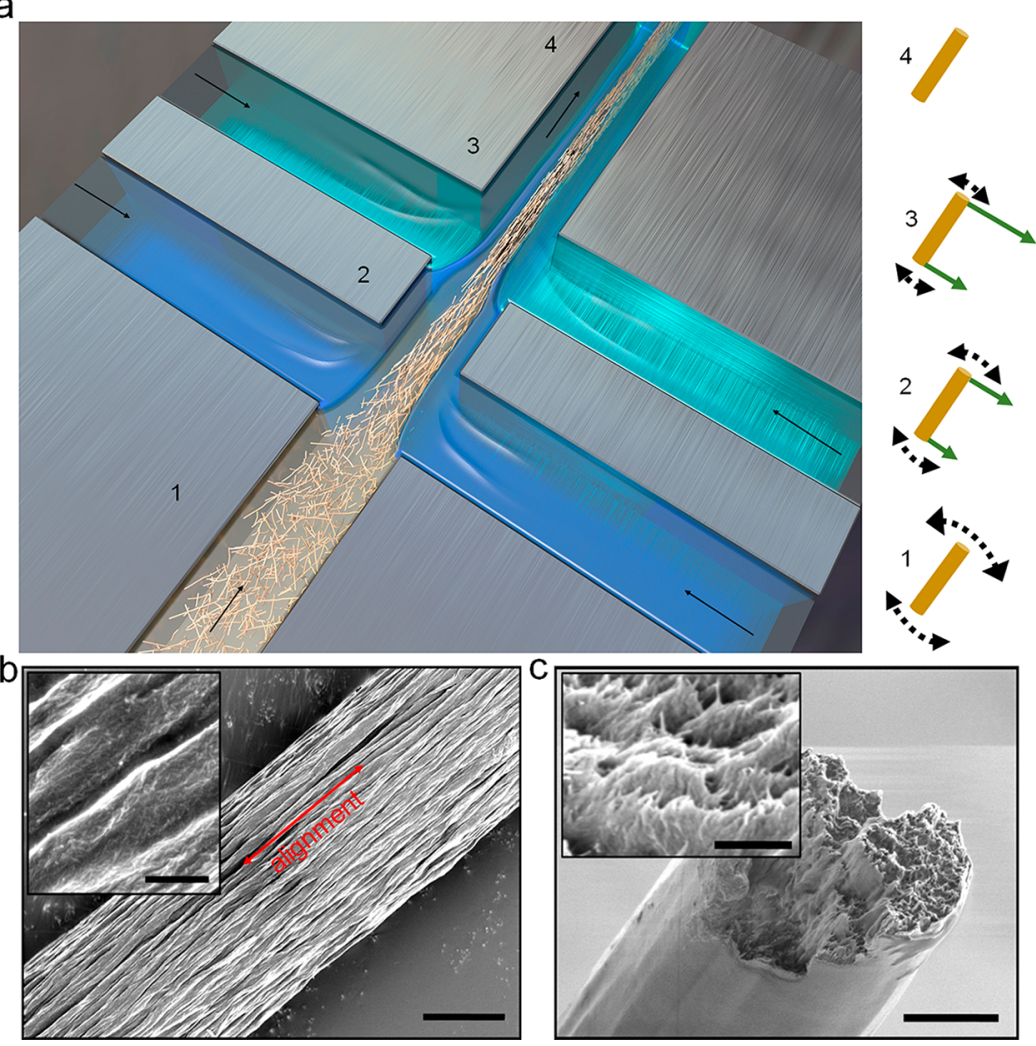

Figure 1. Assembly of nanostructured CNF fibers. (a) Schematic of double flow-focusing channel used for CNF assembly. The CNF suspension is injected in the core flow (light brown color), DI water (blue color) in the first sheath, and acid at low $\mathrm{pH}$ (light green color) in the second sheath flows. Arrows show the flow direction. Hydrodynamic and electrostatic interactions at different positions along the channel are illustrated schematically on the right. Position 1, poor fibril alignment due to Brownian diffusion and electrostatic repulsion (illustrated with the dashed arrows) caused by dissociated carboxyl (-COOH) groups on the fibrils surface. Position 2 hydrodynamically induced alignment (illustrated by solid, green arrow) occurs during acceleration/extension. Position 3 further increase in alignment during acceleration/extension, and in position 4, following the acid addition, Brownian diffusion is minimized due to the transition of CNF suspension to an immobilized volume filling arrested state due to protonation of the $\mathrm{COO}^{-}$groups. For illustration, the relative size of the fibrils has been magnified around $\mathbf{3 0 0}$ times. The use of acid for transforming the free-flowing CNF suspension to a fibrous colloidal glass ensures that the electrostatic repulsions are replaced by van der Waals forces in the protonated carboxyl groups. This complete removal of electrostatic repulsion is not possible with simple electrolytes. ${ }^{22}$ (b) SEM image of the fiber surface, where the dense fibrillar network with well-preserved anisotropic arrangement can be seen. (c) SEM image of the cross section of the fiber, showing the aligned nanofibrils. Scale bars in (b) and (c) are $3 \mu \mathrm{m}$ and insets are $400 \mathrm{~nm}$.

$\mathrm{GPa}) .5,7,8$ Unfortunately, macroscopic materials from these structural components have mechanical properties that are 315 times short of the theoretical and experimentally determined values characteristic of the individual fibrils due to difficulties in the assembly of CNFs into macroscale dense structures, promoting efficient stress transfer between them and inhibiting the occurrence of stochastic defects. ${ }^{3,9}$

Flow-assisted assembly is a promising method for fabricating large, well-ordered edifices of nanoscale objects. ${ }^{10-13}$ However, the colloidal behavior of CNF in liquids is known to be more complicated than that of isotropic nanomaterials, monodispersed nanorods, or carbon nanotubes due to broad distribution of length, process-induced deformations, facile gelation into a disorganized glassy state, and complexity of $\mathrm{CNF}-\mathrm{CNF}$ interactions in different orientations. ${ }^{9,14}$ Hydrodynamic stresses from extensional flows are known to effectively break dense colloidal aggregates and to produce dispersions with steady-state ordering of materials, in contrast to shear flows. ${ }^{15,16}$ Inspired by the architecture of the S2 layers, we here make use of insights into the behavior of nanofibrils under flow and organize them into dense macroscale fibers with in situ-controlled organization that resolve the problems of multiscale stress transfer discussed above..$^{11,17-21}$

\section{RESULTS AND DISCUSSION}

Macroscale fibers from nanoscale CNFs are fabricated by hydrodynamic alignment of the fibrils from a surface-chargecontrolled sol. ${ }^{22}$ In this process, it is vital to align the fibrils in the suspension before "locking" the nanostructure into metastable colloidal glass. This was accomplished using wellestablished fundamentals of extensional flow fields ${ }^{13,23}$ provided by a double flow-focusing channel (Figure 1a). In the core flow, charged CNF fibrils are free to rotate due to electrostatic repulsions and Brownian motion (Figure 1a, position 1), only restrained by fibril-fibril interactions. Note that the electrostatic repulsion caused by the dissociated $\mathrm{COOH}$ groups on the surface of CNFs is much higher than the attractive van der Waals forces at neutral to slightly alkaline $\mathrm{pH}$. The first sheath flow of deionized (DI) water supports electrostatic repulsion and prevents transition into the glass state in contact with channel walls; it also aligns the fibrils toward the flow direction (position 2). ${ }^{13,17}$ Before the alignment is diminished by the Brownian diffusion, the second flow of low $\mathrm{pH}$ acid enhances 
a

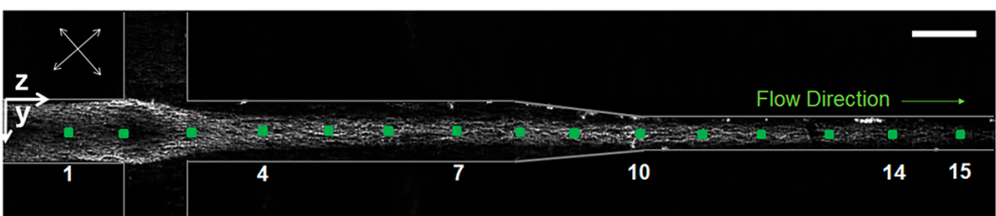

b

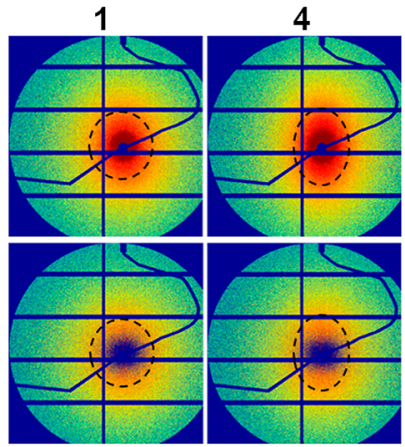

7

10

14
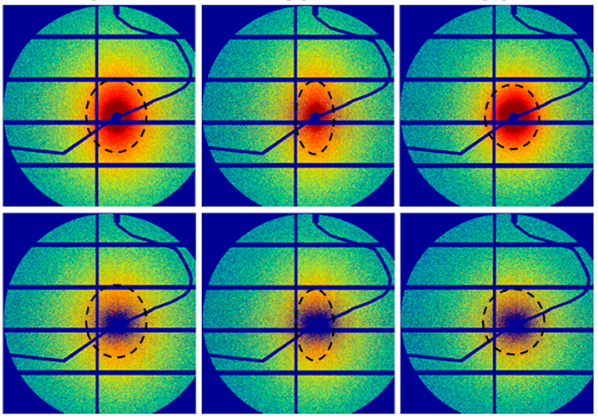

C

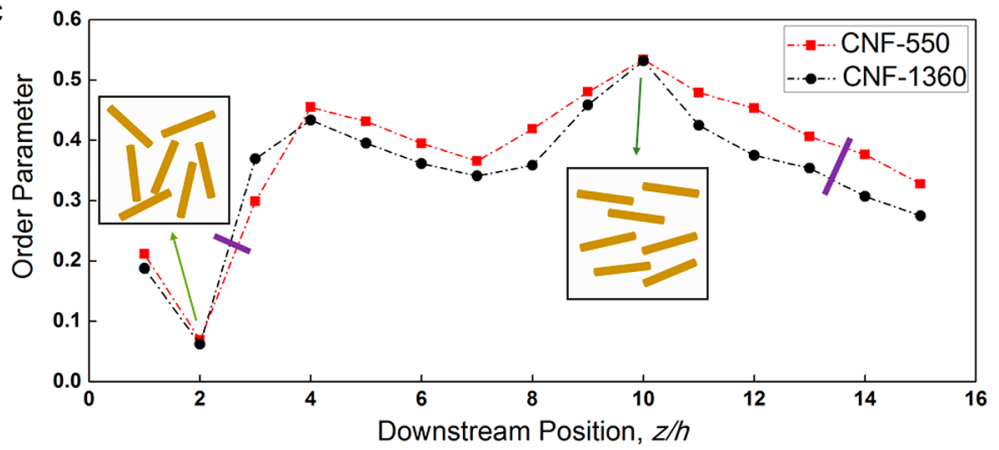

d
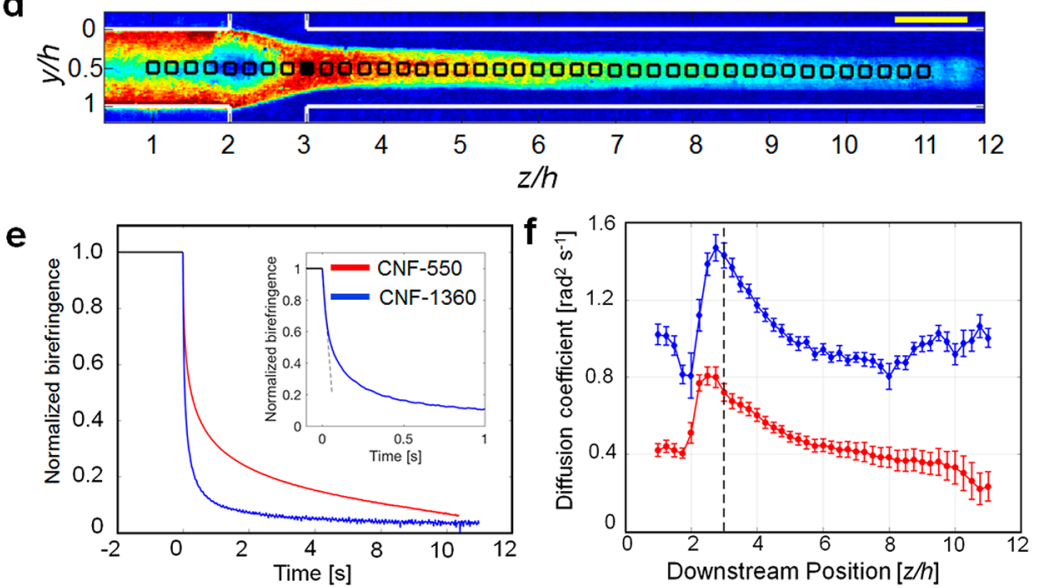

Figure 2. In situ study of the alignment, dealignment and rotary diffusion of fibrils. (a) Microscopy image of the channel used for $\mu$ SAXS measurements, placed between two cross-polarized filters rotated $45^{\circ}$ from the vertical axis (white arrows). White color corresponds to the birefringence signal obtained for the CNF-550 suspension. Numbers represent the positions where in situ measurements were carried out. Scale bar is $1 \mathrm{~mm}$. (b) $\mu$ SAXS scattering diffractograms at different positions along the channel for CNF-550 suspension (top row) and CNF1360 suspension (bottom row). Curved lines represent the beam stopper. (c) Local order parameters calculated from the $\mu$ SAXS scattering diffractograms as a function of downstream position normalized with the channel width $(h)$. (d) Birefringence signal obtained in a single flowfocusing channel for the CNF-550 suspension. Scale bar is $1 \mathrm{~mm}$. The black squares along the center line represent the positions where the $D_{\mathrm{r}}$ values are estimated. (e) Birefringence signal obtained at $z / h=3$ as a function of time when the flow is viciously stopped. The inset shows the initial decay of the birefringence signal for the CNF-1360 suspension. The dashed line indicates the fit of the initial decay to measure the $D_{\mathrm{r}}$. Negative and positive time represents the condition before and after stopping the flow, respectively. (f) $D_{\mathrm{r}}$ as a function of downstream position along the center line. The vertical dashed line shows the position $z / h=3$ from where the birefringence decays are plotted in (e). This position also corresponds to the filled black square at $z / h=3$ in (d). The colors in (e) and (f) correspond to different suspensions: CNF-550 (red) and CNF-1360 (blue).

the fibril alignment (position 3), while reducing the electrostatic repulsion between the fibrils due to protonation of carboxyl $\left(\mathrm{COO}^{-}\right)$groups that allows the supramolecular interactions between CNFs to self-organize the fibrils into a well-packed state with maximized $\mathrm{CNF}-\mathrm{CNF}$ contacts (position 4) (see Figure S1 for further details on the 


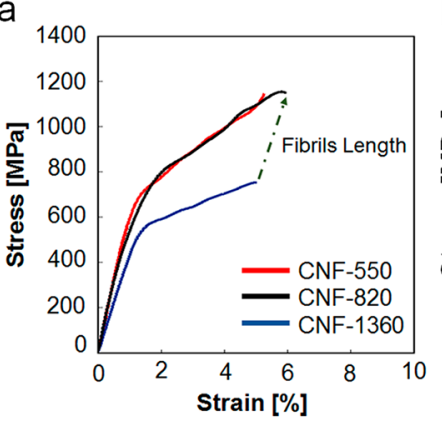

d

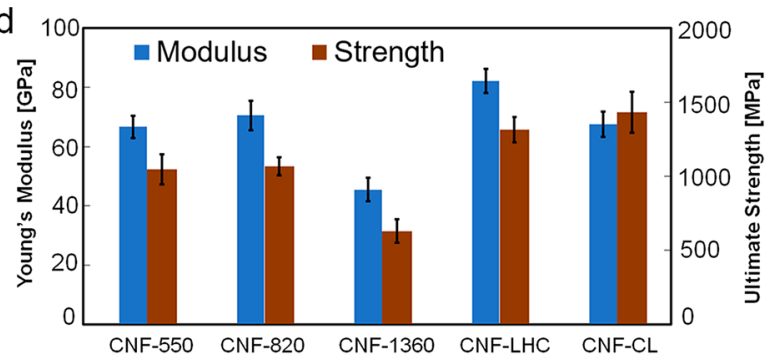

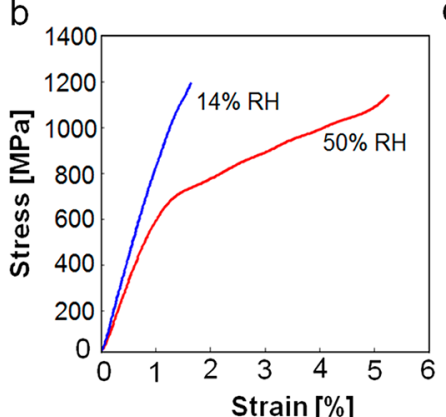

e

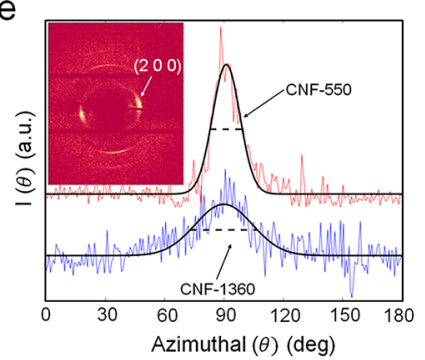

Figure 3. Tensile mechanical properties and nanostructure characterization of the prepared fibers. (a) Stress-strain curves for nanostructured fibers made from fibrils of different lengths indicating their influence on tensile mechanics of CNF fibers prepared from double flow-focusing channel measured at 50\% relative humidity ( $\mathrm{RH})$. Effect of (b) physical (RH) and (c) chemical (cross-linking with BTCA) approaches for tuning the tensile mechanical properties of fibers (prepared from CNF-550 suspension). Plots comparing (d) Young's modulus and ultimate strength of different fibers prepared in this work. LHC and CL stand for low humidity condition and cross-linking, respectively. Error bars correspond to the standard deviation obtained from 10 samples for each case. (e) Azimuthal integration of the (200) scattering plane of the diffractograms for CNF-550 and CNF-1360 samples. Diffractogram corresponding to a CNF-550 fiber is shown in the inset.

experimental setup). The continuous threads obtained from the flow-induced assembly were subsequently held at their ends and air-dried. Characterization of the assembled structure with scanning electron microscopy (SEM) (Figure 1b) sampled in the longitudinal direction showed uniformly sized CNF fibers with dense and near perfectly aligned fibrils without obvious packing defects or voids (Figure S2). Micrographs of fiber cross sections sampled in the transversal direction confirm the dense fibrillar packing and reveal a well-defined layered structure (Figure 1c).

In situ monitoring of CNF assembly in solution was essential for successful adaptation of multiple conditions. Polarization microscopy shows the alignment of fibrils (Figure 2a), with gradually increasing birefringence from randomized suspensions to aligned fibrils. ${ }^{13}$ Concurrently, in situ alignment can be quantified using synchrotron-based microfocus small-angle Xray scattering ( $\mu$ SAXS) (Figure $2 \mathrm{~b}$ ) (see Methods for details). Local order parameters of CNF suspensions with different lengths of the constituting fibrils (CNF-550 of $590 \mathrm{~nm}$ and CNF-1360 of $391 \mathrm{~nm}$, the suffix represent the surface charges in $\mu$ equiv $\mathrm{g}^{-1}$ ) are calculated (Figure $2 \mathrm{c}$ and Figures S3 and S4). An order parameter of 1 represents a fully aligned state of the fibrils (in the direction of fiber preparation), and 0 corresponds to an isotropic fibril distribution.

Initially, the shear from channel walls gave rise to some ordering $(z / h=1)$ in the CNF suspension. At the beginning of the focusing step $(z / h=2)$, the order parameter decreases due to deceleration of the core flow followed by a sudden increase after the focusing due to acceleration $(3 \leq z / h<4)$. Subsequently, the order parameter decreases slightly $(4 \leq z)$ $h<7)$ before increasing again during acceleration in the contraction $(7 \leq z / h<10)$. The decrease in order parameter after the focusing and contraction steps $(4 \leq z / h<7 ; 10 \leq z / h$ $<15$ ) indicates nanofibril relaxation toward isotropy, primarily due to Brownian diffusion. ${ }^{13}$ Substantial differences between the alignment and disorganization behavior of CNF-550 and CNF-1360 suspensions are observed: the shorter the fibrils (CNF-1360), the faster the process of alignment and dirorganization (Figure 2c). This effect is due to the diffusivity based on length distributions of the nanofibrils as given by the rotary diffusion coefficient $\left(D_{\mathrm{r}}\right)$ of CNF-1360, which is twice that of to CNF-550 (Figure 2d-f) (see Methods for details).

Establishing a relationship between fibril characteristics (length, surface charge) and mechanical properties, particularly when fabrication technique involves fibrils under flow induced stretching, is vital to provide the foundation for future rational design of materials with targeted performance maxima. For a comprehensive understanding, we fabricated another set of fibers (CNF-820 with mean fibrils length of $683 \mathrm{~nm}$ ) and compared the mechanical properties of CNF-550, CNF-820, and CNF-1360. The stress-strain curves (Figure 3a) show an initial linear region (pseudoelastic), followed by significant deviation from linearity (plastic region). The "knee" in the curve represents the elastic-plastic transition and is attributed to yielding mechanisms related to sliding of the fibrils. ${ }^{9}$ CNF550 and CNF-820 show similar stress-strain behavior with a modulus and strength of $\sim 70 \mathrm{GPa}$ and $\sim 1200 \mathrm{MPa}$, respectively (Figure $3 \mathrm{a}-\mathrm{d}$ ). This could be due to the similar mean length of the constituent fibrils and indicates that strength and stiffness of the prepared fibers are relatively independent of fibril surface charge. ${ }^{24}$ This is further supported by a significant decrease in strength $(630 \mathrm{MPa})$ by reducing fibril length to $391 \mathrm{~nm}$ (CNF-1360). Moreover, the lower modulus of CNF-1360 (45 GPa) is due to the relatively low fibril orientation, as verified by wide-angle $\mathrm{X}$-ray scattering (WAXS) (Figure 3e). The orientation index for CNF-550 fibers is 0.92 (order parameter $=0.70$ ), whereas CNF-1360 fibers show 0.83 (order parameter $=0.53$ ). In general, the stiffest 
materials tend to be the strongest if provided with defect-free structure and strong interfaces to ensure adequate load transfer and cohesion within the material. ${ }^{2,3}$ The strength and stiffness of our fibers vividly demonstrate the importance of connectivity and bonding at the fibril-fibril interfaces facilitating multiscale stress transfer.

To further extend the property range of these materials, we evaluated different approaches based on physical (varying the ambient condition) and chemical (covalent cross-linking of the fibrils) strategies. With CNF-550 fibers conditioned at 14\% RH for a period of $40 \mathrm{~h}$ before testing, CNF-LHC exhibited a modulus and strength values as high as $82 \pm 4 \mathrm{GPa}$ and $1320 \pm$ $85 \mathrm{MPa}$, respectively (Figure 3b). Similar strength (1320 \pm $56.5 \mathrm{MPa}$ ) is calculated from the Weibull analysis, ${ }^{25,26}$ with a $m$ value of 28.9 , indicating very few defects inside the fibers. As one might partially expect from previous studies of bioinspired nanocomposites, strengthening of interfibrillar interactions and removal of water molecules from the fibril surface resulted in marked improvement of fiber mechanics with respect to other CNF composites but also led to embrittlement and low failure strain. At high humidity, water acts as a plasticizer and allows extended elastic deformations, thus reducing the stiffness and increasing the strain. ${ }^{27}$ To reduce the humidity effect, the CNF550 fibers were cross-linked by 1,2,3,4-butane tetracarboxylic acid (BTCA) that was used to neutralize the suspensions during the flow-based assembly of the fibers. BTCA creates covalent bridges between $\mathrm{CNF}$ fibrils, ${ }^{28}$ replicating to some degree the cross-links between cellulose and lignin/hemicellulose. ${ }^{6}$ The average strength of cross-linked fiber (CNF-CL) tested at $50 \% \mathrm{RH}$ increased to $1430 \mathrm{MPa}$ (highest measured value of $1570 \mathrm{MPa}$ ) with negligible change in modulus (Figure $3 \mathrm{c}, \mathrm{d})$. Chemical cross-linking introduces covalent bonds between the fibrils, which improves the connectivity and stress transfer. ${ }^{29}$

Additionally, the high strain to failure obtained for the uncross-linked CNF fibers $(\sim 6 \%)$ is rather uncommon for highly oriented structures. Structural changes and interfibrillar molecular interactions were further investigated by cyclic loading-unloading tests in the post-yield regime (Figure S5). The post-yield modulus may decrease due to the formation of cracks or increase due to reorientation of the fibrils (as for the case of random-in-plane CNF network) along the test direction. ${ }^{30}$ Interestingly, the Young's modulus in the present case remains unchanged upon unloading at post-yield strain values (see Methods for details). This suggests a lack of structural changes, and the mechanisms of plastic deformation must involve reformable secondary bonds as in the case of stick-slip. ${ }^{31}$ This was further verified by molecular dynamics (MD) simulations (Figure S6), where the number of hydrogen bonds (per unit area) remains constant during the relative sliding of the fibrils. ${ }^{32}$ Upon cross-linking the CNF fibers, the plastic deformation is substantially reduced, and the stressstrain curve becomes relatively linear as the secondary interactions are replaced by covalent bonds (Figure 3c). Although interfibrillar interactions are dominated by the relatively weak hydrogen bonds and van der Waals forces, the highly aligned state of the fibrils amplifies their effects due to collective synergy of molecular interlocking, leading to stiffening and effective energy dissipating mechanisms (stickslip and molecular zip-up). ${ }^{31,33}$

It is worth highlighting that even the properties of the "weaker" fiber (CNF-1360) have previously been unachievable for CNF fibers fabricated with other approaches. ${ }^{34-36}$ Hence, there is a strong and profound benefit of exploiting extensional flow fields for alignment and assembly of nanofibrils (or elongated particles, in general), giving a fresh insight for the proper selection in future high-performance fibers by getting closer to the theoretical limit. Further, the orientation index values for highly aligned CNF-550 reported in this work are only slightly higher than those reported for CNF-based macrofibers fabricated with other approaches. ${ }^{9,27,37,38}$ However, the up to 6 times higher strength of our fibers (Table S1) indicates that even in fibers with aligned nanoscale building blocks, interfaces and interactions play a key role in controlling the mechanical properties.

The increased strength and stiffness values of the CNF fibers made by the double flow-focusing method make it feasible to use them for numerous load-bearing applications. ${ }^{39}$ The materials data chart (Figure 4) demonstrates that CNF fibers

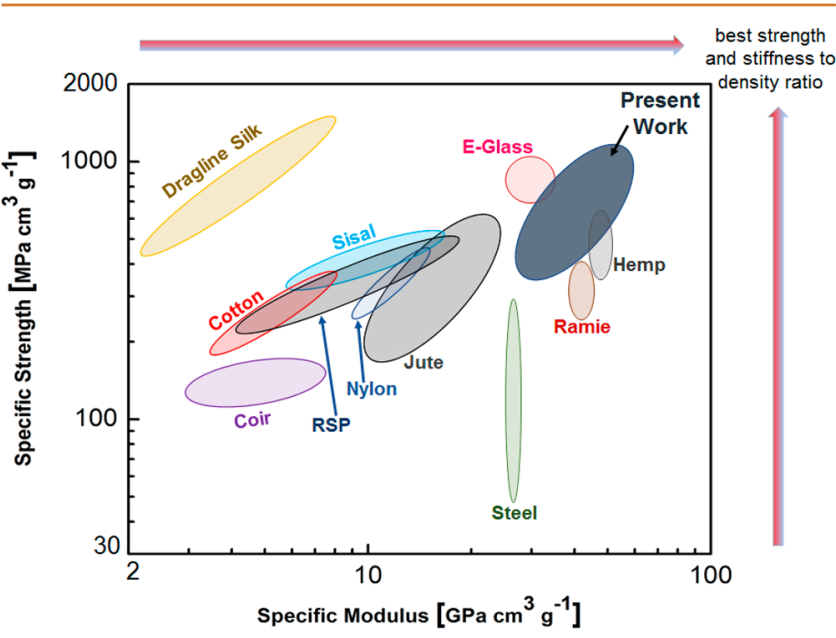

Figure 4. Tensile mechanical properties of bio-based and selected synthetic fiber materials. Overview of specific ultimate strength versus specific Young's modulus for a range of bio-based materials, steel, and E-glass from the non-bio-based resources. The region of fibers fabricated in the present work is shown in dark gray. Details on the mechanical properties' data drawn for different materials are included in the Supporting Information (Table S2).

have strength and stiffness that markedly exceed all natural and commercial bio-based materials. This includes natural wood pulp fibers with high orientation of nanoscale crystalline planes, free from damage and natural imperfections (see Methods for details), and wet-spun high aspect ratio nanocelluloses that recently attracted considerable interest. ${ }^{24,34,35}$ The latter methods as well as other fiber-drawing techniques used in the past for carbon nanotubes are shear dominated, ${ }^{40}$ where a large nozzle diameter is used and thick fibers $(25-200 \mu \mathrm{m})$ are formed. Consequently, a fibrillar network of lower density and more random orientation of the fibrils are formed, which compromises load transfer between nanoscale building blocks in the macroscopic material. Fibers fabricated through assembly of CNF with these approaches never managed to reach stiffness and strength beyond $35 \mathrm{GPa}$ and $600 \mathrm{MPa}$, respectively. ${ }^{9,13}$

The comparison with the properties of dragline silk, the gold standards for lightweight biopolymers, is also revealing. Notable, the CNF fibers outperform dragline silk by a factor of 8 in terms of stiffness with strengths on the same level (Figure 2). Furthermore, the specific strength of our CNF fibers now exceeds metals, alloys, and silica-based E-glass fibers $^{13}$ (Table S2). Interestingly, the strongest CNF fibers are 
1.2-1.5 times stronger than wet-spun carbon nanotubes and graphene fibers. ${ }^{40,41}$ Note needs to be made that theoretical strength of crystalline cellulose is orders of magnitude lower than that of carbon nanotubes and graphene. This is possibly a limiting factor because the specific strength and stiffness of the highly oriented CNF fibers are still lower than aramid, ultrahigh molecular weight polyethylene, and carbon fibers (Figure S7). The current results highlight the central role of processing strategies and associated fundamental parameters (e.g., building blocks size, interactions, and network formation) on realizing the true potential of nanoscale building blocks.

\section{CONCLUSIONS AND OUTLOOK}

Flow-based assembly is the promising route for scalable fabrication of structural materials with a highly ordered arrangement of nanoscale building blocks such as fibrils, polymers, nanotubes, and nanorods. ${ }^{11,12,42,43}$ Exceptional mechanical properties of the reported fibers from nanoscale cellulose support this conclusion. Throughout processing, the tuning of interparticle interactions is imperative with respect to their mobility and structural integrity. The physical dimensions of the particles are the key, as diffusion due to Brownian motion and physical interactions are central at the nanoscale. Given the time scales relevant to this study, which is on the order of subseconds to seconds (see Methods for details), effects on the orientation of individual nanofibrils due to rotary diffusion caused by Brownian motion are significantly faster than all other time scales. ${ }^{44-46}$ However, the time scales for rotary diffusion of fibrils in semidilute suspensions are longer than what would be expected from Brownian motion for an individual fibril, which allows flow-field-based alignment to achieve nanostructure control. Furthermore, an extensional flow field will align fibrils without intermittent flipping and rotation, as is the case for shear where alignment is a timeaveraged quantity (Figure S8). ${ }^{15,47,48}$

When concentration increases, fibril-fibril interactions not only impede mobility but also provide necessary structural integrity during the assembly. The degree of interaction can be estimated by the crowding factor $(N)$ and the average number of contact points per fibril $\left(\bar{n}_{\mathrm{c}}\right){ }^{49,50}$ The crowding factor is defined as

$$
N=\frac{2}{3} \phi\left(\frac{l}{d}\right)^{2}=\frac{2}{3} \phi A^{2}
$$

where $\phi$ is the volumetric concentration and the aspect ratio (A) equals $l / d$, where $l$ and $d$ are length and diameter of the fibrils, respectively. Furthermore, the average number of contact points $\left(\bar{n}_{\mathrm{c}}\right)$ for an individual fibril is given by

$$
\bar{n}_{\mathrm{c}}=\frac{3}{A} N
$$

where $\bar{n}_{\mathrm{c}}$ is dependent on the orientation distribution. ${ }^{51}$ Structural integrity between the fibrils is given by force transfer at interfibrillar contact points by physiochemical interactions (electrostatic interactions, van der Waals interactions, or chemical cross-linking) and mechanical interlocking through contact point normal forces and friction governed by fibril bending stiffness. ${ }^{52}$ In the former case, connectivity is given by $\bar{n}_{\mathrm{c}}>2$ (otherwise, there can be only a single string of individual fibrils), whereas mechanical interlocking requires $\bar{n}_{\mathrm{c}} \geq 3$ and implies that elastic energy is stored in the network.
The propensity for this mechanical interlocking is readily described by $N$, where $N<1$ implies no interlocking and a threshold referred to as the colloidal glass crowding factor, indicating a transition to a more interconnected system, being essential for network formation. The thresholds depend on the "connectivity" and "rigidity" as predicted by effective medium and percolation theories. The implication with respect to current results is that mechanical interlocking between the fibrils is present during all stages of flow-focusing. This is evident from the measured times scales of rotary diffusion (Figure 2e) that are an order of magnitude longer than Brownian motion of an individual fibril. Although $\bar{n}_{\mathrm{c}}$ varies from 0.4 to 0.8 in our experiments, which ideally would allow free rotation, crystalline and noncrystalline domains provide partly flexible fibrils with sufficient rigidity, causing mechanical interlocking even in the suspension state due to contact point friction, even though the electrostatic repulsions induced from the surface charges provide mobility. ${ }^{51}$ This is further evident from the behavior of cellulose nanocrystals that are unable to form a strong enough network with the same approach as they lack flexibility. The suspended "loose" network of fibrils allows uniform rearrangement and alignment under the extensional flow, which cannot be achieved by only shear action localized in different regions of the flow field. To fabricate nanostructured fibers with high degree of orientation, the conditions (such as length of fibrils and concentration) must be tuned such that there is a restraint on Brownian motion by mechanical interlocking without impeding the mobility that will promote the flow-field-induced alignment. Furthermore, there is room for further optimization of mechanial properties of the biobased cellulosic materials by tuning the architecture or interparticle interactions to achieve and potentially redefine the theoretical limit.

\section{METHODS}

CNF Preparation. CNF suspensions were prepared from chemically bleached wood fibers (a mixture of $60 \%$ Norwegian spruce and $40 \%$ Scots pine, provided by Domsjö Fabriker AB, Sweden). The relative glucose content of the pulp was $>96 \%$. Wood pulp fibers were chemically treated with a 2,2,6,6-tetramethylpiperidinyl-1-oxyl (TEMPO)-mediated oxidation reactions as reported elsewhere. ${ }^{53}$ CNF with surface charge densities in the medium to high range $\left(550-1360 \mu\right.$ equiv $\left.\mathrm{g}^{-1}\right)$ were obtained by varying the reaction time and/or conditions. For the CNF with surface charge densities of 550 and $820 \mu$ equiv $\mathrm{g}^{-1}$, cellulose pulp fibers $(1 \mathrm{~g})$ were suspended in 0.05 $\mathrm{M}$ sodium phosphate $\left(\mathrm{Na}_{3} \mathrm{PO}_{4}\right)$ buffer $(90 \mathrm{~mL}, \mathrm{pH} 6.8)$ by dissolving TEMPO (16 mg, $0.1 \mathrm{mmol})$ and sodium chlorite $\left(\mathrm{NaClO}_{2}\right)(80 \%$, $1.13 \mathrm{~g}, 10 \mathrm{mmol})$. The $2 \mathrm{M}$ sodium hypochlorite $(\mathrm{NaClO})$ solution $(0.5 \mathrm{~mL})$ after dilution to $0.1 \mathrm{M}$ with the $0.05 \mathrm{M} \mathrm{Na}_{3} \mathrm{PO}_{4}$ buffer was added to the suspension. The suspensions were stirred at $500 \mathrm{rpm}$ for a designated time ( 2 and $48 \mathrm{~h}$ for surface charge density of 550 and $820 \mu$ equiv $\mathrm{g}^{-1}$, respectively). For the CNF with surface charge density of $1360 \mu$ equiv $\mathrm{g}^{-1}$, wood pulp fibers were suspended at a concentration of $1 \mathrm{wt} \%$ in DI water with the addition of TEMPO (16 $\mathrm{mg} \mathrm{g}^{-1}$ cellulose) and sodium bromide $(\mathrm{NaBr})\left(100 \mathrm{mg} \mathrm{g}^{-1}\right.$ cellulose). $\mathrm{NaClO}\left(5.0 \mathrm{mmol} \mathrm{g}^{-1}\right)$ was added dropwise to the suspension with vigorous stirring. The $\mathrm{pH}$ of the suspension was maintained at a constant value of 10 with the addition of $0.1 \mathrm{M}$ sodium hydroxide $(\mathrm{NaOH})$ solution until no change in $\mathrm{pH}$ was observed. The TEMPO-oxidized pulp fibers were washed thoroughly with DI water by filtration. Aqueous suspensions of the fibers were passed through a high-pressure homogenizer after the chemical pretreatment. At the end of this step, CNF suspensions, with a concentration of $>5 \mathrm{~g} \mathrm{~L}^{-1}$ were obtained. The gel-like suspensions were diluted by adding DI water and mixed thoroughly using a mechanical mixer $(12000 \mathrm{rpm}$ for 10 $\mathrm{min}$, Ultra Turrax, IKA, Germany) followed by the sonication (10 min, 
Sonics Vibracell, USA). The diluted suspensions were then centrifuged at $5000 \mathrm{rpm}$ for $60 \mathrm{~min}$, and the precipitates were removed. The supernatants were then used for further studies. The dry content of the suspensions was determined by gravimetric analysis. All the chemicals were purchased from Sigma-Aldrich (unless otherwise stated) and used without further purification. All the fibers were prepared at a CNF concentration of $3 \mathrm{~g} \mathrm{~L}^{-1}$.

Fibril Characterization. The lengths of $\sim 250 \mathrm{CNFs}$ (for each charge density) using transmission electron microscopy (TEM) (JEOL JEM-1400 TEM) at an accelerating voltage of $120 \mathrm{kV}$ were measured (Figure S3). Ruby camera was used to acquire the images following "systematic, uniform, random" rule to avoid bias. The sample was deposited on a carbon-coated copper grid treated with glow discharge and stained with $2 \%$ uranyl acetate solution prior to the observation.

The height of $\sim 150$ CNFs (for each charge density) using atomic force microscopy (AFM) (MultiMode 8, Bruker, Santa Barbara, CA, USA) (Figure S4) was measured. Silicon wafers (Addison Engineering Inc., San José, CA, USA) were oxidized at $1000{ }^{\circ} \mathrm{C}$ for $1 \mathrm{~h}$ for the formation of a silica layer. The wafers were washed with ethanol and milli-Q followed by drying and treatment in the plasma chamber (PCD 002, Harrick Scientific Corp., Ossining, NY, USA) for 5 min to make the surface hydrophilic. Wafers at the size of $5 \times 5 \mathrm{~mm}$ were dipped in the CNF suspensions and left to dry in air at room temperature.

Degree of polymerization (DP) was calculated from the intrinsic viscosity data using the protocol reported earlier. ${ }^{30}$ The measurements were performed on the untreated pulp and CNF suspensions with copper ethylenediamine as solvent. Dry weight contents of $150 \mathrm{mg}$ were suspended in $25 \mathrm{~mL}$ of water with copper bolts and mixed until the particles were no longer visible, whereupon $25 \mathrm{~mL}$ of copper ethylenediamine was added, and the samples were shaken until the cellulose had dissolved. The temperature was controlled by submersion into a $25{ }^{\circ} \mathrm{C}$ water bath, controlled by a thermostat for $30 \mathrm{~min}$. The obtained DP values for the untreated pulp and CNF with surface charge densities of 550, 820, and $1360 \mu$ equiv $\mathrm{g}^{-1}$ were 1121 , 582,533 , and 271 , respectively.

Conductometric titration ${ }^{54}$ was used to determine the carboxylate contents of CNFs. Suspensions $(100 \mathrm{~mL}, 0.1 \mathrm{wt} \%)$ were used, and the $\mathrm{pH}$ was adjusted to 2.5 with $0.1 \mathrm{M} \mathrm{HCl}$. The suspensions were then titrated with $0.01 \mathrm{M}$ standardized $\mathrm{NaOH}$ by adding $0.2 \mathrm{~mL}$ aliquots in $60 \mathrm{~s}$ intervals until the $\mathrm{pH}$ reached 11 , and the conductivity was monitored with a benchtop meter (FE20 FiveEasy, Mettler-Toledo). The titration curves displayed the presence of strong and weak acid groups, where the amount of strong acid linked with the added $\mathrm{HCl}$ and that of weak acid with the carboxyl contents.

The degree of crystallinity calculated from $\mathrm{CP} / \mathrm{MAS}{ }^{13} \mathrm{C}$ NMR is 26 $\pm 1,30 \pm 1,38 \pm 1$, and $27 \pm 1 \%$ for the untreated pulp, CNF-550, CNF-820, and CNF-1360, respectively. CP/MAS ${ }^{13} \mathrm{C}$ NMR is crosspolarization magic angle spinning carbon-13 nuclear magnetic resonance spectra. All samples were packed uniformly in a zirconium oxide rotor. The $\mathrm{CP} / \mathrm{MAS}{ }^{13} \mathrm{C}$ NMR spectra were recorded in a Bruker Avance III AQS 400 SB instrument operating at $9.4 \mathrm{~T}$. All measurements were carried out at $295( \pm 1) \mathrm{K}$ with a magic angle spinning rate of $10 \mathrm{kHz}$. A $4 \mathrm{~mm}$ double air-bearing probe was used. Data acquisition was performed using a cross-polarization pulse sequence (i.e., a $2.95 \mu$ s proton, $90^{\circ}$ pulse, and $800 \mu$ s ramped (100$50 \%$ ) falling contact pulse, with a $2.5 \mathrm{~s}$ delay between repetitions). A SPINAL64 pulse sequence was used for ${ }^{1} \mathrm{H}$ decoupling. The Hartmann-Hahn matching procedure was based on glycine. The chemical shift scale was calibrated to the TMS scale (tetramethylsilane, $\left(\mathrm{CH}_{3}\right)_{4} \mathrm{Si}$ ) by assigning the data point of maximum intensity in the $\alpha$ glycine carbonyl signal to a shift of $176.03 \mathrm{ppm}$. A total of 4096 transients were recorded on each sample, leading to an acquisition time of about $3 \mathrm{~h}$. The software for spectral fitting was developed at Innventia $\mathrm{AB}$ and is based on a Levenberg-Marquardt algorithm. ${ }^{55}$ All computations were based on integrated signal intensities obtained from spectral fitting. ${ }^{56}$ The errors given for parameters obtained from the fitting procedure are the standard error of the mean with respect to the quality of the fit.
Flow Setup. The flow setup consists of three syringe pumps (WPI, Al-4000), one double flow-focusing channel and a water bath (Figure S1). The syringe pumps transfer CNF suspension in the core flow, DI water in the first sheath, and acid at low $\mathrm{pH}$ in the second sheath flows of the channel. Flow rates of core flow, first, and second sheath flows correspond to $4.1,4.4$, and $24.6 \mathrm{~mL} \mathrm{~h}^{-1}$, respectively. The channel was milled into $1 \mathrm{~mm}$ thick stainless-steel plate and sealed between two plexiglas plates. Two aluminum plates were placed on either side and screwed together to prevent the leakage. The width of channels was 1 $\mathrm{mm}$. The outlet of the channel was submerged in a DI water bath. The hydrogel threads of CNF were picked from the water bath with the help of tweezers followed by air drying at room temperature for at least $2 \mathrm{~h}$. Solid CNF fibers were obtained after drying the hydrogel threads.

Fibril Cross-Linking. 1,2,3,4-Butanetetracarboxylic acid was dissolved in DI water until the $\mathrm{pH}$ reached 2.4. Sodium hypophosphite (SHP), 50\% by weight of the BTCA, was added to the solution. This solution was used in the second sheath flow instead of $\mathrm{HCl}$ for suspension to gel transition. Gel/colloidal glass threads from the water bath were dried in an oven at $105^{\circ}$ for $1 \mathrm{~h}$. Cross-linked CNF-550 fibers were placed in the conditioning room at $50 \% \mathrm{RH}$ for $24 \mathrm{~h}$ before the tensile testing.

In Situ $\mu$ SAXS Measurements. $\mu$ SAXS experiments were performed at P03 beamline at PETRA III storage ring at DESY, Hamburg. A slightly modified channel geometry was used to study the in situ behavior of fibrils under the dynamic flow conditions, where the second focusing step was mimicked by a contraction step, and the width of the channel after the contraction is reduced to $0.5 \mathrm{~mm}$. However, both channels work on the same principle of extensional flow fields. Experiments were carried out in the absence of gelation due to the long exposure times and limited access to the synchrotron. The flow rates were the same as used for the fiber formation in the core flow and first sheath flow. The channel was sandwiched between Kapton windows instead of plexiglass plates that were used for the fiber fabrication experiments. The measurements were performed in the transmission geometry with an X-ray wavelength of $\lambda=0.96 \AA$ and sample-to-detector distance of $6950 \mathrm{~mm}$. Beam size was $20 \times 10 \mu \mathrm{m}^{2}$ (horizontal $\times$ vertical), and a single-photon counting detector (Pilatus $300 \mathrm{k}$ by Dectris, Switzerland) with a pixel size of $172 \times 172 \mu \mathrm{m}^{2}$ was used to monitor the scattering diffractograms.

Order parameters to quantify the CNF alignment were calculated from the $\mu$ SAXS scattering diffractograms based on a procedure similar to that described elsewhere. ${ }^{13,57,58}$ In brief, scattering diffractograms were transformed into the diffractogram with scattering vector $(q ; q=$ $4 \pi \sin (\varphi) / \lambda$, where $\varphi$ is scattering angle) and azimuthal angle $(\theta)$ as coordinates. The background intensities (with flow of only DI water) were removed from the scattering intensities obtained with the CNF. The zero-level intensity is determined by assuming that, at the most aligned position in the channel, there are no fibrils aligned perpendicularly to the flow direction. Therefore, the intensity at $\theta=$ 0 or $\pi$ for this position is subtracted from all the distributions. The final intensity (orientation) distributions were averaged between $0.1<$ $q<0.5 \mathrm{~nm}^{-1}$ for each azimuthal angle.

The alignment of CNF was quantified by converting the orientation distribution in terms of order parameter $(S)$, defined as

$$
S=\left\langle\frac{3}{2} \cos ^{2} \theta-\frac{1}{2}\right\rangle
$$

where $\theta$ is the azimuthal angle in a diffractogram. Expanding the average gives

$$
S=\int_{0}^{\pi} I(\theta)\left(\frac{3}{2} \cos ^{2} \theta-\frac{1}{2}\right) \sin \theta \mathrm{d} \theta
$$

which is normalized according to

$$
\int_{0}^{\pi} I(\theta) \sin \theta d \theta=1
$$

where $I(\theta)$ is the intensity distribution averaged along examined $q$ value for each azimuthal angle. 
Fiber Characterization. Samples for SEM were prepared by sputtering the fibers surface with a $5 \mathrm{~nm}$ thin gold-palladium layer (Gressington Instruments Ltd., UK). Surface structure analysis was performed by using a field emission scanning electron microscope (Hitachi S-4800, Japan) operated at an acceleration voltage of $1 \mathrm{kV}$.

For information about the fiber bulk structure, samples were embedded in epoxy-embedding medium (Sigma-Aldrich) and polymerized overnight at $60{ }^{\circ} \mathrm{C}$. Ultrathin sections $(80 \mathrm{~nm})$ were cut with a diamond knife (Diatome, Switzerland) on a Leica ultracut UCT (Leica Microsystems, Germany) ultramicrotome and placed on 75 mesh Formvar coated copper grids (Electron Microscopy Sciences). Images were taken at $120 \mathrm{kV}$ with a JEOL 1400plus TEM equipped with Ruby camera (both from JEOL, Japan).

The tensile tests were performed on Instron E100 instrument equipped with a $5 \mathrm{~N}$ load cell. Fibers were conditioned at room temperature $\left(23{ }^{\circ} \mathrm{C}\right)$ and $50 \% \mathrm{RH}$ (unless otherwise stated) for at least $40 \mathrm{~h}$ prior to testing. The dimensions were measured by an optical microscope (Nikon Japan- Eclipse Ni-E) and further crosschecked with SEM for a few random samples. Individual CNF fibers were uniform in cross section throughout the length; however, the diameter may differ between the different fibers. Typical diameter of the CNF fibers is around $6.8 \pm 0.9 \mu \mathrm{m}$. The fiber ends were glued on paper (Figure S9), and the whole assembly was mounted on the tensile test instrument and clamped between the grips. The vertical part of the paper strip was then cut from the center, so that the fibers were only held by the grips at both ends. The span length was 9-12 $\mathrm{mm}$, and measurements were carried out at a crosshead speed of 0.5 $\mathrm{mm} \mathrm{min}{ }^{-1}$. The cross section of the fibers was assumed to be circular. Density of the nanostructured CNF fibers is assumed to be $1500 \mathrm{~kg}$ $\mathrm{m}^{-3}$.

WAXS measurements were carried out at PETRA III storage ring (P03 beamline) at DESY, Hamburg. ${ }^{57}$ Three samples were measured for each case. Measurements were performed at an X-ray wavelength $\lambda$ $=0.96 \AA$, and the sample-to-detector distance was set to $71 \mathrm{~mm}$. The beam size was $6 \times 14 \mu \mathrm{m}^{2}$ (horizontal $\times$ vertical). The scattering diffractograms were recorded using a Pilatus $300-\mathrm{k}$ detector (Dectris) with a pixel size of $172 \times 172 \mu \mathrm{m}^{2}$. Intensity distribution profiles were used to calculate the order parameter $(S)$ and orientation index $\left(f_{\mathrm{c}}\right)$, according to the equation ${ }^{54}$

$$
f_{\mathrm{c}}=\frac{180^{\circ}-\mathrm{fwhm}}{180^{\circ}}
$$

where fwhm is the full width at half-maximum of the azimuthal profiles.

Diffusion Measurements. Experiments for rotational diffusivity were performed in a single step flow-focusing channel, with a square cross section of $1 \mathrm{~mm}$ (Figure S10). The plexiglass plates were replaced by thick COC films (Tekni-plex $8007 \mathrm{X}-04$ ). The flow rate ratio used was the same as used in the core and first sheath flows for the fiber fabrication. The collective anisotropy of fibrils is visualized using polarized optical microscopy (POM) technique, where the channel is illuminated orthogonally by a laser beam. Before entering the channel, the beam first passes through a polarizer, oriented at $45^{\circ}$ with respect to the channel downstream direction $z$. Then, after the channel, the light encounters a second polarizer, oriented at $90^{\circ}$ with respect to the first one. Finally, the light is collected by a camera (Mako U, Allied Vision) at 100 fps. This method used the birefringence characteristics of CNF suspensions, where the light intensity recorded by the camera depends on the birefringence (i.e., the alignment). The intensity collected by the camera is equal to

$$
I=I_{0} \sin ^{2}(\Delta \gamma)
$$

with $\Delta \gamma=\frac{2 \pi e}{\lambda} \Delta n$, the phase shift. Here, $e$ is the thickness of the sample, $\lambda$ is the wavelength of the laser light, and $\Delta n$ is the optical index difference created by the alignment of the CNF, due to birefringence. $I_{0}$ is the laser-intensity-dependent unknown constant. In our case, the phase shift $\Delta \gamma$ is much smaller than 1 . This leads to $I \sim$ $I_{0} \Delta \gamma^{2} \sim I_{0} \Delta n^{2}$. Thus, the birefringence is proportional to the square root of the intensity recorded by the camera. As $I_{0}$ is unknown, one can have access only to the relative birefringence, normalized to start at 1 before we stop the flow

$$
\Delta n(t)=\sqrt{\frac{I(t)}{I(t<0)}}
$$

To observe the decay behavior of aligned CNFs, the flow is stopped viciously by using four solenoid-driven slider valves (Takasago Electric, Inc.), one being set on each branch of the flow-focusing channel. The time to stop the flow was relatively smaller compared to the acquisition rate. Once the flow is stopped, the birefringence decay was recorded for $10 \mathrm{~s}$. Examples are shown in Figure 2e.

Time Scales Controlling the Assembly Process. In the double flow-focusing channel, the diameter of the CNF suspension jet decreases significantly at each focusing step. After the first one, the jet reaches a diameter $\varepsilon_{1}$ approximately equal to $0.5 \mathrm{~h}$. After the second focusing point, the diameter $\varepsilon_{2}$ is around $0.2 \mathrm{~h}$. From this, one can estimate the velocities along the centerline. (a) Before focusing: $v_{0} \sim$ $2 Q_{c} / h^{2}$, which is $2.3 \mathrm{~mm} \mathrm{~s}^{-1}$, assuming a Poiseuille flow. (b) After the first focusing step and before the second one: $v_{1} \sim 4 Q_{c} / \pi \varepsilon_{1}^{2}$, which is $5.8 \mathrm{~mm} \mathrm{~s}^{-1}$, assuming a plug flow. (c) After the second focusing step: $v_{2} \sim 4 Q_{c} / \pi \varepsilon_{2}^{2}$, which is $37.1 \mathrm{~mm} \mathrm{~s}^{-1}$, assuming a plug flow.

The alignment of the fibrils is achieved through the acceleration at the first and second focusing steps. If this acceleration is effective during a distance $2 \mathrm{~h}$ from the focusing point, the alignment time scales for the first and second focusing steps are given by

$$
t_{\text {align1 }} \sim 2 h /\left(v_{1}-v_{0}\right) \sim 0.58 \mathrm{~s} \text { and } t_{\text {align2 }} \sim 2 h /\left(v_{2}-v_{1}\right) \sim 0.06 \mathrm{~s}
$$

Moreover, the convective time scale, relative to the transport of the CNF gel thread from the second focusing point to the water bath, is $t_{\text {conv }} \sim L / v_{2} \sim 1.2 \mathrm{~s}$, where $L$ is the distance between these two points $(45 \mathrm{~mm})$

To form a gel network from the CNF suspensions, ions from the acid must diffuse inside the CNF jet. The time scale associated with this diffusion process can be estimated using the diffusion equation in cylindrical coordinates, as reported by Håkansson and co-workers. ${ }^{13}$ For $\mathrm{HCl}$ in water, the diffusion coefficient is $D_{\text {ion }}=3 \times 10^{-9} \mathrm{~m}^{2} \mathrm{~s}^{-1}$. The minimum ion concentration necessary to gel the CNF suspension is estimated by solving the diffusion equation up to $t=t_{\text {conv }}$, with an initial ion concentration of $3 \mathrm{mM}$. Indeed, we have noticed that this ion concentration was the minimum concentration able to gel the entire thread before it reaches the water bath. At $t=t_{\text {conv }}$, the ion concentration in the center of the thread is about $0.63 \mathrm{mM}$, now defined as the gelling concentration. The diffusion equation is then solved again with an initial ion concentration of $10 \mathrm{mM}$, corresponding to $\mathrm{pH} 2 \mathrm{HCl}$, that is, to the conditions of the filament fabrication. The time scale $t_{\text {ion }}$ for the ion concentration to reach the gelling concentration of $0.63 \mathrm{mM}$ in the center of the thread has been found to be equal to $0.68 \mathrm{~s}$.

In the ungelled state, there will be a Brownian rearrangement of the fibrils toward isotropy. This is a diffusion-dominated process, where within $0.68 \mathrm{~s}\left(t_{\text {ion }}\right)$, at least $60 \%$ of the alignment for CNF-550 is lost due to rotary diffusion. This loss in alignment within this time scale is more than $90 \%$ for CNF-1360 (Figure 2e).

Molecular Dynamics Simulation. Two 36-chain fibrils in a diamond cross-section shape, with DP 30 and 60, respectively, were constructed based on atomic coordinates from the X-ray and neutron diffraction of highly crystalline cellulose samples. ${ }^{59}$ The hydrogen bonding network A was selected as it is more energetically stable than the other one (pattern $\mathrm{B}$ ) and was considered as the main pattern within core of cellulose. ${ }^{60}$ Both the fibrils have 110 and $1-10$ planes exposed on the surface. About $1 / 3$ of exterior hydroxymethyl groups along every surface chain were "selected" to be oxidized into $\mathrm{COOH}$ group, representing the $600 \mu$ equiv $\mathrm{g}^{-1}$ surface charge density to mimic the fibrils used for fiber fabrication.

Molecular dynamics simulations were performed by using GROMACS package, ${ }^{61}$ version 2016.3, and the GLYCAM06 force field. ${ }^{62}$ VMD package ${ }^{63}$ was used to visual the trajectory file and to create graphs. All the structures contained in the system was optimized by energy minimization first using steepest descents and followed 
conjugate gradient methods. The initial structures of the two finite fibrils were then individually relaxed for both $50 \mathrm{~ns}$ before merging them into the same simulation box. Other details were identical to the study done by Chen and co-workers. ${ }^{64}$

The slippage between cellulose fibrils was modeled using the pulling code implemented in GROMACS. Initially, two fibrils were both aligned in the $z$-axis of a simulation box that has the dimensions of 10 $\times 10 \times 40\left(\mathrm{~nm}^{3}\right)$. The DP-30 fibril was placed on the top of DP-60 one, faced by the 110 surface to each other in a parallel manner and was distant by $1 \mathrm{~nm}$. MD simulation was performed to allow the unforced aggregation of two fibrils. Until the system reaches equilibrium, a pulling rate of $0.0005 \mathrm{~nm} \mathrm{ps}^{-1}$ and a pulling force of $10000 \mathrm{~kJ} \mathrm{~mol}^{-1} \mathrm{~nm}^{-1}$, parallel to the fiber direction ( $z$-axis), are applied in the reducing group of DP-30 chain, whereas the core region of the bottom fibril was freeze. Hydrogen bonds are analyzed by using gmx h-bonds tool. Only the hydrogen bonds formed by hydroxyl groups were considered. Those from $\mathrm{C}-\mathrm{H}$ groups are ignored. A donor-acceptor distance of $<0.35 \mathrm{~nm}$ and the angle hydrogen donoracceptor $<30^{\circ}$ are used for the criteria of hydrogen bonds determination.

Interfibrillar Interactions and Deformation Mechanism. The high strain to failure of highly oriented structures is rather uncommon. The post-yield plastic deformation mechanisms were further investigated by cyclic loading-unloading tests, which reveal details of structural changes and interfibrillar molecular interactions involved (Figure S5). Interestingly, the fiber integrity is completely recovered upon unloading at post-yield strain values. Several micro- and nanocomposites show a decrease in modulus due to the damages in the form of cracks at reinforcement-matrix interface. ${ }^{30}$ Studies on random-in-plane CNF networks show increased modulus post yield point, ${ }^{30}$ which is attributed to the reorientation of the nanofibrils along the test direction. However, unchanged modulus in the present case suggests a lack of further change in orientation (due to the highly aligned state of the nanofibrils in the CNF fibers) and the mechanisms of plastic deformation must involve reformable secondary bonds as in the case of stick-slip. ${ }^{31}$ The existence of stick-slip is further confirmed by MD simulations (Figure S6), where the number of hydrogen bonds (per unit area) remains constant during the relative sliding of the fibrils. ${ }^{32}$ These results are further supported by the tensile test data obtained from covalently cross-linked CNF fibers, where plastic deformation is substantially reduced after the secondary interactions start being replaced by the covalent bonds (Figure 3c). Keckes and co-workers ${ }^{31}$ reported similar behavior (unchanged stiffness) in the plastic range of individual wood cells, corresponding to a "stick-slip" mechanism. The proposed mechanism involves breakage and reformation of new "unspecified" bonds at molecular and supramolecular level, possibly mediated by the polymers (hemicelluloses) present along the cellulose microfibrils.

Rotational Diffusivity of the Nanofibrils. The diffusivity of the nanofibrils is studied by determining the rotary diffusion coefficient $\left(D_{\mathrm{r}}\right)$ to quantify the relaxation of the fibrils alignment. Figure $2 \mathrm{~d}$ shows the birefringence signal obtained for CNF-550 suspension using the POM technique. When the flow is turned off, the advection of nanofibrils by the flow is stopped, and fibrils start to dealign due to rotary diffusion. Accordingly, the birefringence signal decays toward 0 . Examples of such relaxations at $z / h=3$, when the signal is normalized by the initial birefringence, are shown in Figure 2e. This position is marked by the filled black square in Figure 2d. The relaxation of the birefringence is expected to be an exponential decay, with a characteristic time equal to $1 / 6 D_{\mathrm{r}}{ }^{45}$ However, the decays (Figure 2e) cannot be fully fitted by an exponential function. Indeed, the decays show several time scales but for our purposes, the $D_{r}$ are estimated using the initial decay, ${ }^{65}$ between 0 and $0.1 \mathrm{~s}$ (dotted line), as shown in the inset.

Figure $2 \mathrm{f}$ shows $D_{\mathrm{r}}$ as a function of the downstream position in the channel. The two curves exhibit the same general behavior: a large increase in the focusing region $(z / h$ between 2 and 3$)$ followed by a smaller but longer decrease. Moreover, the $D_{\mathrm{r}}$ values of the CNF-1360 suspension are approximately 2 times larger than the one for the CNF550 suspension. This discrepancy is likely owing to the difference in length distributions of the fibrils (Figure S3) as the $D_{\mathrm{r}}$ values are strongly dependent on the length of the particles. ${ }^{66}$ As the $D_{\mathrm{r}}$ values for CNF-1360 suspension are larger, the corresponding fibers exhibited lower alignment of fibrils (Figure 3e). These results along with the SAXS and WAXS measurements clarify the reason behind lower strength of fibers obtained with CNF-1360 suspension.

Mechanical Properties of Defect-Free Wood Pulp Fibers. Natural wood pulp fiber with high orientation of nanoscale crystalline planes, free from damage and natural imperfections, has a highest reported strength of $\sim 1600 \mathrm{MPa}$ (stiffness unknown). ${ }^{67}$ It is worthwhile mentioning that this wood fiber possessed structural integrity of a tree provided by nature through centuries of evolution, where CNFs are embedded in soft amorphous matrices of natural binders (hemicelluloses and lignins) that are used to distribute the stress and reduce the defects. Interestingly, our laboratory-designed $\mathrm{CNF}$ fibers reach the strength equal to the highest reported values for a wood pulp fiber with stiffness on the order of $\sim 70-90 \mathrm{GPa}$.

\section{ASSOCIATED CONTENT}

\section{S Supporting Information}

The Supporting Information is available free of charge on the ACS Publications website at DOI: 10.1021/acsnano.8b01084.

Detailed illustration of the assembly process, TEM images of the nanostructured fibers, TEM images and length characterization of the CNFs, AFM image and height characterization of the CNFs, cyclic loadingunloading test, molecular dynamics simulations, illustration on the arrangement of fibrils while processing through extensional and shear-based flow systems, schematic of the POM experimental setup, tables for the comparison of mechanical properties' data and associated references (PDF)

\section{AUTHOR INFORMATION}

\section{Corresponding Author}

*E-mail: dansod@kth.se.

ORCID

Farhan Ansari: 0000-0001-7870-6327

Christophe Brouzet: 0000-0003-3131-3942

Lars Wågberg: 0000-0001-8622-0386

Nicholas A. Kotov: 0000-0002-6864-5804

L. Daniel Söderberg: 0000-0003-3737-0091

\section{Author Contributions}

N.M., F.A., F.L., L.W., N.A.K., and L.D.S. conceived the idea including the development and design of methodology. L.D.S. supervised the study. N.M. and F.A. prepared and characterized CNF suspensions. N.M. fabricated the fibers and evaluated fiber properties and deformation mechanisms with assistance from F.A. and L.D.S. N.M., K.G.V., S.V.R., F.L., and L.D.S. performed the SAXS and WAXS experiments. C.B. performed the rotational diffusion experiments with assistance from N.M. N.M., F.A., K.G.V., C.B., and L.D.S. analyzed the data. P.C. carried out molecular dynamics simulations. P.T.L. performed and analyzed the NMR measurements. N.M., F.A., N.A.K., and L.D.S. wrote the manuscript with input from other co-authors.

\section{Notes}

The authors declare no competing financial interest.

\section{ACKNOWLEDGMENTS}

This research has been funded by the Knut and Alice Wallenberg Foundation through Wallenberg Wood Science Center at KTH. The authors are grateful to Yoshiharu Nishiyama and Lilian Medina for scientific discussions. Mehmet 
Yilmaz, Ayaka Kamada, Tomas Rosén, Wiebke Ohm, and Michaela Salajkova are thanked for experimental assistance. Authors also acknowledge the kind support of Irene Linares Arregui from the Solid Mechanics Department at KTH with the tensile tests.

\section{REFERENCES}

(1) Munch, E.; Launey, M. E.; Alsem, D. H.; Saiz, E.; Tomsia, A. P.; Ritchie, R. O. Tough, Bio-Inspired Hybrid Materials. Science 2008, $322,1516-1520$.

(2) Barthelat, F.; Yin, Z.; Buehler, M. J. Structure and Mechanics of Interfaces in Biological Materials. Nat. Rev. Mater. 2016, 1, 16007.

(3) Wegst, U. G.; Bai, H.; Saiz, E.; Tomsia, A. P.; Ritchie, R. O. Bioinspired Structural Materials. Nat. Mater. 2015, 14, 23-36.

(4) Podsiadlo, P.; Kaushik, A. K.; Arruda, E. M.; Waas, A. M.; Shim, B. S.; Xu, J.; Nandivada, H.; Pumplin, B. G.; Lahann, J.; Ramamoorthy, A.; Kotov, N. A. Ultrastrong and Stiff Layered Polymer Nanocomposites. Science 2007, 318, 80-83.

(5) Gibson, L. J. The Hierarchical Structure and Mechanics of Plant Materials. J. R. Soc., Interface 2012, 9, 2749-66.

(6) Atalla, R. H.; Agarwal, U. P. Raman Microbe Evidence for Lignin Orientation in the Cell Walls of Native Woody Tissue. Science 1985, 227, 636-639.

(7) Ashby, M.; Gibson, L.; Wegst, U.; Olive, R. The Mechanical Properties of Natural Materials. I. Material Property Charts. Proc. $R$. Soc. London, Ser. A 1995, 450, 123-140.

(8) Saito, T.; Kuramae, R.; Wohlert, J.; Berglund, L. A.; Isogai, A. An Ultrastrong Nanofibrillar Biomaterial: The Strength of Single Cellulose Nanofibrils Revealed via Sonication-Induced Fragmentation. Biomacromolecules 2013, 14, 248-253.

(9) Benítez, A.; Walther, A. Cellulose Nanofibril Nanopapers and Bioinspired Nanocomposites: A Review to Understand the Mechanical Property Space. J. Mater. Chem. A 2017, 5, 16003-16024.

(10) Yu, G.; Cao, A.; Lieber, C. M. Large-Area Blown Bubble Films of Aligned Nanowires and Carbon Nanotubes. Nat. Nanotechnol. 2007, 2, 372-377.

(11) Kamada, A.; Mittal, N.; Söderberg, L. D.; Ingverud, T.; Ohm, W.; Roth, S. V.; Lundell, F.; Lendel, C. Flow-Assisted Assembly of Nanostructured Protein Microfibers. Proc. Natl. Acad. Sci. U. S. A. 2017, 114, 1232-1237.

(12) Davis, V. A.; Parra-Vasquez, A. N. G.; Green, M. J.; Rai, P. K.; Behabtu, N.; Prieto, V.; Booker, R. D.; Schmidt, J.; Kesselman, E.; Zhou, W.; Fan, H.; Adams, W. W.; Hauge, R. H.; Fischer, J. E.; Cohen, Y.; Talmon, Y.; Smalley, R. E.; Pasquali, M. True Solutions of SingleWalled Carbon Nanotubes for Assembly into Macroscopic Materials. Nat. Nanotechnol. 2009, 4, 830-834.

(13) Håkansson, K. M.; Fall, A. B.; Lundell, F.; Yu, S.; Krywka, C.; Roth, S. V.; Santoro, G.; Kvick, M.; Wittberg, L. P.; Wågberg, L. Hydrodynamic Alignment and Assembly of Nanofibrils Resulting in Strong Cellulose Filaments. Nat. Commun. 2014, 5, 4018.

(14) Usov, I.; Nyström, G.; Adamcik, J.; Handschin, S.; Schütz, C.; Fall, A.; Bergström, L.; Mezzenga, R. Understanding Nanocellulose Chirality and Structure-Properties Relationship at the Single Fibril Level. Nat. Commun. 2015, 6, 7564.

(15) Folgar, F.; Tucker, C. L. Orientation Behavior of Fibers in Concentrated Suspensions. J. Reinf. Plast. Compos. 1984, 3, 98-119.

(16) Zaccone, A.; Soos, M.; Lattuada, M.; Wu, H.; Bäbler, M. U.; Morbidelli, M. Breakup of Dense Colloidal Aggregates Under Hydrodynamic Stresses. Phys. Rev. E 2009, 79, 061401.

(17) Trebbin, M.; Steinhauser, D.; Perlich, J.; Buffet, A.; Roth, S. V.; Zimmermann, W.; Thiele, J.; Förster, S. Anisotropic Particles Align Perpendicular to the Flow Direction in Narrow Microchannels. Proc. Natl. Acad. Sci. U. S. A. 2013, 110, 6706-6711.

(18) Lutz-Bueno, V.; Zhao, J.; Mezzenga, R.; Pfohl, T.; Fischer, P.; Liebi, M. Scanning-SAXS of Microfluidic Flows: Nanostructural Mapping of Soft Matter. Lab Chip 2016, 16, 4028-4035.

(19) Rogers, S. S.; Venema, P.; Sagis, L. M. C.; van der Linden, E.; Donald, A. M. Measuring the Length Distribution of a Fibril System: A
Flow Birefringence Technique Applied to Amyloid Fibrils. Macromolecules 2005, 38, 2948-2958.

(20) Smith, D. E.; Babcock, H. P.; Chu, S. Single-Polymer Dynamics in Steady Shear Flow. Science 1999, 283, 1724-1727.

(21) Perkins, T. T.; Smith, D. E.; Chu, S. Single Polymer Dynamics in an Elongational Flow. Science 1997, 276, 2016-2021.

(22) Fall, A. B.; Lindström, S. B.; Sundman, O.; Ödberg, L.; Wågberg, L. Colloidal Stability of Aqueous Nanofibrillated Cellulose Dispersions. Langmuir 2011, 27, 11332-11338.

(23) Nunes, J. K.; Tsai, S. S. H.; Wan, J.; Stone, H. A. Dripping and Jetting in Microfluidic Multiphase Flows Applied to Particle and Fiber Synthesis. J. Phys. D: Appl. Phys. 2013, 46, 114002.

(24) Lundahl, M. J.; Klar, V.; Wang, L.; Ago, M.; Rojas, O. J. Spinning of Cellulose Nanofibrils into Filaments: A Review. Ind. Eng. Chem. Res. 2017, 56, 8-19.

(25) Lee, C.; Wei, X.; Kysar, J. W.; Hone, J. Measurement of the Elastic Properties and Intrinsic Strength of Monolayer Graphene. Science 2008, 321, 385-388.

(26) Naito, K.; Tanaka, Y.; Yang, J.-M.; Kagawa, Y. Tensile Properties of Ultrahigh Strength PAN-Based, Ultrahigh Modulus Pitch-Based and High Ductility Pitch-Based Carbon Fibers. Carbon 2008, 46, 189-195.

(27) Benítez, A. J.; Torres-Rendon, J.; Poutanen, M.; Walther, A. Humidity and Multiscale Structure Govern Mechanical Properties and Deformation Modes in Films of Native Cellulose Nanofibrils. Biomacromolecules 2013, 14, 4497-4506.

(28) Nyström, G.; Marais, A.; Karabulut, E.; Wågberg, L.; Cui, Y.; Hamedi, M. M. Self Assembled Three-Dimensional and Compressible Interdigitated Thin-Film Supercapacitors and Batteries. Nat. Commun. 2015, 6, 7259.

(29) Mamedov, A. A.; Kotov, N. A.; Prato, M.; Guldi, D. M.; Wicksted, J. P.; Hirsch, A. Molecular Design of Strong Single-Wall Carbon Nanotube/Polyelectrolyte Multilayer Composites. Nat. Mater. 2002, 1, 190-194.

(30) Henriksson, M.; Berglund, L. A.; Isaksson, P.; Lindström, T.; Nishino, T. Cellulose Nanopaper Structures of High Toughness. Biomacromolecules 2008, 9, 1579-1585.

(31) Keckes, J.; Burgert, I.; Fruhmann, K.; Muller, M.; Kolln, K.; Hamilton, M.; Burghammer, M.; Roth, S. V.; Stanzl-Tschegg, S.; Fratzl, P. Cell-Wall Recovery After Irreversible Deformation of Wood. Nat. Mater. 2003, 2, 810-813.

(32) Zhu, H.; Zhu, S.; Jia, Z.; Parvinian, S.; Li, Y.; Vaaland, O.; Hu, L.; Li, T. Anomalous Scaling Law of Strength and Toughness of Cellulose Nanopaper. Proc. Natl. Acad. Sci. U. S. A. 2015, 112, 89718976.

(33) Keten, S.; Xu, Z.; Ihle, B.; Buehler, M. J. Nanoconfinement Controls Stiffness, Strength and Mechanical Toughness of [Beta] Sheet Crystals in Silk. Nat. Mater. 2010, 9, 359-367.

(34) Walther, A.; Timonen, J. V.; Díez, I.; Laukkanen, A.; Ikkala, O. Multifunctional High-Performance Biofibers Based on Wet-Extrusion of Renewable Native Cellulose Nanofibrils. Adv. Mater. 2011, 23, 2924-2928.

(35) Iwamoto, S.; Isogai, A.; Iwata, T. Structure and Mechanical Properties of Wet-Spun Fibers Made from Natural Cellulose Nanofibers. Biomacromolecules 2011, 12, 831-836.

(36) Hooshmand, S.; Aitomäki, Y.; Norberg, N.; Mathew, A. P.; Oksman, K. Dry-Spun Single-Filament Fibers Comprising Solely Cellulose Nanofibers from Bioresidue. ACS Appl. Mater. Interfaces 2015, 7, 13022-13028.

(37) Mittal, N.; Jansson, R.; Widhe, M.; Benselfelt, T.; Håkansson, K. M. O.; Lundell, F.; Hedhammar, M.; Söderberg, L. D. Ultrastrong and Bioactive Nanostructured Bio-Based Composites. ACS Nano 2017, 11, $5148-5159$.

(38) Lundahl, M. J.; Cunha, A. G.; Rojo, E.; Papageorgiou, A. C.; Rautkari, L.; Arboleda, J. C.; Rojas, O. J. Strength and Water Interactions of Cellulose I Filaments Wet-Spun from Cellulose Nanofibril Hydrogels. Sci. Rep. 2016, 6, 30695.

(39) Meyers, M. A.; Chen, P.-Y.; Lin, A. Y.-M.; Seki, Y. Biological Materials: Structure and Mechanical Properties. Prog. Mater. Sci. 2008, 53, 1-206. 
(40) Behabtu, N.; Young, C. C.; Tsentalovich, D. E.; Kleinerman, O.; Wang, X.; Ma, A. W. K.; Bengio, E. A.; Ter Waarbeek, R. F.; De Jong, J. J.; Hoogerwerf, R. E.; Fairchild, S. B.; Ferguson, J. B.; Maruyama, B.; Kono, J.; Talmon, Y.; Cohen, Y.; Otto, M. J.; Pasquali, M. Strong, Light, Multifunctional Fibers of Carbon Nanotubes with Ultrahigh Conductivity. Science 2013, 339, 182-186.

(41) Xin, G.; Yao, T.; Sun, H.; Scott, S. M.; Shao, D.; Wang, G.; Lian, J. Highly Thermally Conductive and Mechanically Strong Graphene Fibers. Science 2015, 349, 1083-1087.

(42) Dzenis, Y. Spinning Continuous Fibers for Nanotechnology. Science 2004, 304, 1917-1919.

(43) Vigolo, B.; Pénicaud, A.; Coulon, C.; Sauder, C.; Pailler, R.; Journet, C.; Bernier, P.; Poulin, P. Macroscopic Fibers and Ribbons of Oriented Carbon Nanotubes. Science 2000, 290, 1331-1334.

(44) Brenner, H. Rheology of a Dilute Suspension of Axisymmetric Brownian Particles. Int. J. Multiphase Flow 1974, 1, 195-341.

(45) Doi, M.; Edwards, S. F. Dynamics of Rod-Like Macromolecules in Concentrated Solution. Part 1. J. Chem. Soc., Faraday Trans. 2 1978, 74, 560-570.

(46) Tao, Y. G.; Den Otter, W.; Padding, J.; Dhont, J.; Briels, W. Brownian Dynamics Simulations of the Self-And Collective Rotational Diffusion Coefficients of Rigid Long Thin Rods. J. Chem. Phys. 2005, $122,244903$.

(47) Stover, C. A.; Koch, D. L.; Cohen, C. Observations of Fibre Orientation in Simple Shear Flow of Semi-Dilute Suspensions. J. Fluid Mech. 1992, 238, 277-296.

(48) Shaqfeh, E. S.; Koch, D. L. Orientational Dispersion of Fibers in Extensional Flows. Phys. Fluids A 1990, 2, 1077-1093.

(49) Celzard, A.; Fierro, V.; Kerekes, R. Flocculation of Cellulose Fibres: New Comparison of Crowding Factor With Percolation And Effective-Medium Theories. Cellulose 2009, 16, 983.

(50) Geng, L.; Mittal, N.; Zhan, C.; Ansari, F.; Sharma, P. R.; Peng, X.; Hsiao, B. S.; Söderberg, L. D. Understanding the Mechanistic Behavior of Highly Charged Cellulose Nanofibers in Aqueous Systems. Macromolecules 2018, 51, 1498-1506.

(51) Martoïa, F.; Dumont, P.; Orgéas, L.; Belgacem, M.; Putaux, J. L. Micro-Mechanics of Electrostatically Stabilized Suspensions of Cellulose Nanofibrils Under Steady State Shear Flow. Soft Matter 2016, 12, 1721-1735.

(52) Schmid, C. F.; Klingenberg, D. J. Mechanical Flocculation in Flowing Fiber Suspensions. Phys. Rev. Lett. 2000, 84, 290.

(53) Isogai, A.; Saito, T.; Fukuzumi, H. TEMPO-Oxidized Cellulose Nanofibers. Nanoscale 2011, 3, 71-85.

(54) Tang, H.; Butchosa, N.; Zhou, Q. A Transparent, Hazy, and Strong Macroscopic Ribbon of Oriented Cellulose Nanofibrils Bearing Poly(ethylene glycol). Adv. Mater. 2015, 27, 2070-2076.

(55) Larsson, P. T.; Wickholm, K.; Iversen, T. A CP/MAS13C NMR Investigation of Molecular Ordering in Celluloses. Carbohydr. Res. 1997, 302, 19-25.

(56) Wickholm, K.; Larsson, P. T.; Iversen, T. Assignment of NonCrystalline Forms in Cellulose I by CP/MAS $13 \mathrm{C}$ NMR Spectroscopy. Carbohydr. Res. 1998, 312, 123-129.

(57) Buffet, A.; Rothkirch, A.; Döhrmann, R.; Körstgens, V.; Abul Kashem, M. M.; Perlich, J.; Herzog, G.; Schwartzkopf, M.; Gehrke, R.; Müller-Buschbaum, P.; Roth, S. V. P03, The Microfocus and Nanofocus X-ray Scattering (MiNaXS) Beamline of The PETRA III Storage Ring: The Microfocus Endstation. J. Synchrotron Radiat. 2012, 19, 647-653.

(58) Van Gurp, M. The Use of Rotation Matrices in the Mathematical Description of Molecular Orientations in Polymers. Colloid Polym. Sci. 1995, 273, 607-625.

(59) Nishiyama, Y.; Langan, P.; Chanzy, H. Crystal Structure and Hydrogen-Bonding System in Cellulose $\mathrm{I} \beta$ from Synchrotron X-ray and Neutron Fiber Diffraction. J. Am. Chem. Soc. 2002, 124, 90749082.

(60) Nishiyama, Y. Structure and Properties of the Cellulose Microfibril. J. Wood Sci. 2009, 55, 241-249.

(61) Hess, B.; Kutzner, C.; Van Der Spoel, D.; Lindahl, E. GROMACS 4: Algorithms for Highly Efficient, Load-Balanced, and
Scalable Molecular Simulation. J. Chem. Theory Comput. 2008, 4, 435447.

(62) Kirschner, K. N.; Yongye, A. B.; Tschampel, S. M.; GonzálezOuteiriño, J.; Daniels, C. R.; Foley, B. L.; Woods, R. J. GLYCAM06: A Generalizable Biomolecular Force Field. Carbohydrates. J. Comput. Chem. 2008, 29, 622-655.

(63) Humphrey, W.; Dalke, A.; Schulten, K. VMD: Visual Molecular Dynamics. J. Mol. Graphics 1996, 14, 33-38.

(64) Chen, P.; Ogawa, Y.; Nishiyama, Y.; Ismail, A. E.; Mazeau, K. Linear, Non-Linear and Plastic Bending Deformation of Cellulose Nanocrystals. Phys. Chem. Chem. Phys. 2016, 18, 19880-19887.

(65) Rosén, T. Angular Dynamics of Non-Spherical Particles in Linear Flows Related to Production of Biobased Materials. Doctoral Thesis, Comprehensive Summary, KTH Royal Institute of Technology, Stockholm, 2016.

(66) Doi, M.; Edwards, S. F. The Theory of Polymer Dynamics; Oxford University Press, 1988.

(67) El-Hosseiny, F.; Page, D. H. The Mechanical Properties of Single Wood Pulp Fibres: Theories of Strength. Fibre Sci. Technol. 1975, 8, 21-31. 